\title{
Do Programmer Pairs make different Mistakes than Solo Programmers?
}

\author{
Matthias M. Müller \\ Fakultät für Informatik \\ Universität Karlsruhe \\ Am Fasanengarten 5, 76131 Karlsruhe \\ muellerm@ira.uka.de
}

\begin{abstract}
Objective: Comparison of program defects caused by programmer pairs and solo developers.

Design: Analysis of programs developed during two counter balanced experiments.

Setting: Programming lab at University.

Experimental Units: 42 programs developed by computer science students participating in an extreme programming lab course.

Main Outcome Measures: Programmer pairs make as many algorithmic mistakes but fewer expression mistakes than solo programmers.

Results: The second result is significant on the 5 percent level.

Conclusions: For simple problems, pair programming seems to lead to fewer mistakes than solo programming .
\end{abstract}

\section{INTRODUCTION}

Pair programming has been subject of several controlled experiments in the last years. However, the results draw a rather divergent picture of the productivity of pair programming. While some studies $[9,13]$ report a reduction of development time using pair programming other studies [8] report that there is no benefit. When we look at the defect reduction capabilities, we also see a divergent picture. Some studies report on a reduced defect density caused by pair programming $[13,11]$ while others do not [8]. And finally, pair programming seems to be comparable to partner programming [2] and solo programming with reviews [5, 7].

None of the above studies investigated the impact of the complexity of the task on the productivity of pair programming. It is reported that pair programming is good for "simple problems in simple code" [3]. However, not every code is simple and problems exist that are not easy to solve regardless of the methodology used [4]. On the other hand, programmer pairs form a special kind of group and groups have been studied by the social sciences for a long time. The social science developed the notion of eureka problems. This kind of problems have the characteristic that an individual who solves it will ordinarily feel quite certain that he is right [10, p. 23]. The particularity of eureka problems is that the probability that the group outcome is right depends on the probability that an individual who was able to solve the problem is part of that group.

In this study, we investigated the mistakes programmer pairs and solo programmers made in a sequence of experiments $[5,7]$. Our analysis shows that on the one hand pairs have difficulties with hard problems but on the other hand pairs expose the characteristics of groups for eureka type problems.

\section{DESCRIPTION OF THE DATA SET}

The presented data was collected during two controlled experiments at the University of Karlsruhe which were held during the summer lectures 2002 (Exp02) and 2003 (Exp03). The experiments investigated the question whether solo programming with an additional review phase is comparable to pair programming. Participants were 38 students who took part in an Extreme Programming lab course [6]. The experiments had a counter balanced design. Thus, the 
participants had to solve two problems: Shuffle Puzzle and Polynomial. And each problem had to be solved with a different implementation method: pair programming or solo programming with review. The programming language was Java. Table 1 lists the groups' task order, group sizes, and the number of available data points. To sum up, we have 10 data points for PP.Shu, 11 data

TABLE 1: Task order and available data points for each group ( $\mathrm{PP}=$ pair programming, $\mathrm{So}=\mathrm{solo}$, Shu=Shuffle Puzzle, Pol=Polynomial).

\begin{tabular}{c|cccccc} 
& & & \multicolumn{2}{c}{ Group Sizes } & \multicolumn{2}{c}{ Data Pts. } \\
Group & 1. Task & 2. Task & Exp02 & Exp03 & PP & Solo \\
\hline 1 & PP.Shu & So.Pol & 6 & 4 & 5 & 6 \\
2 & PP.Pol & So.Shu & 4 & 4 & 4 & 4 \\
3 & So.Shu & PP.Pol & 6 & 4 & 5 & 7 \\
4 & So.Pol & PP.Shu & 4 & 6 & 5 & 6 \\
\hline Overall & & & 20 & 18 & 19 & 23
\end{tabular}

points for So.Shu, 9 data points for PP.Pol, and 12 data points for So.Pol.

The procedure for each task was divided into two phases: the implementation phase and an acceptance test phase. During implementation, the participants implemented a solution for the problem until they claimed to be done. Afterwards, they entered the acceptance test phase where they had to rework on remaining errors. This study analyses the defects of the program versions right after the implementation phase.

For a comprehensive description of the experiment procedures, students' programming experience, and division of students into groups the reader is pointed to [5] for Exp02 or to [7] for both experiments. The remaining part of this section focuses on the algorithmic structure of the Shuffle Puzzle and Polynomial tasks and a short description of the defects found.

\subsection{Shuffle Puzzle}

The first task dealt with shuffle puzzles, or as some like to call it, with sliding block puzzles. The problem was to find a minimal solution for a given shuffle puzzle within a given number of moves and to list the moves, if a solution exists. The participants had to add the method findMoves to the basic class ShufflePuzzle. Solving a shuffle puzzle requires implementing a backtracking algorithm using either depth-first or breadth-first search. The task description contained no algorithmic hint for a possible solution. Twenty (20) of the 21 analysed solutions used a depth-first search.

Figure 1 shows the depth-first search part of a possible solution. The code in lines 2 to 4 makes use that the search does not exceed the maximum search depth. The maximum search depth is an input parameter for the implementation. The actual search depth is tracked in lines 5 and 17. The for-loop of lines 6 to 16 iterates through all possible moves. In general, there exist four possible directions in which pieces in a shuffle puzzle can be moved. However, the task description prohibited specifically that a list of moves to a solution must not contain two identical shuffle puzzles. Thus, the method allowedMoves checks the moves performed and prevents a solution from thrashing, for example from moving one piece up and down in two subsequent moves. The moves are applied to the puzzle in line 7 and undone in line 15 . The lines 8 to 12 keep track of possible solutions and store the solution if it is shorter than the shortest solution found so far. The recursive descent is performed in line 15. To ensure that the participants implemented the above described functionality of allowedMoves, the search had to finish within 15 seconds.

Table 2 lists all defects found in the Shuffle Puzzle implementations. The numbers in braces point to a line in the algorithm outlined by Figure 1. The classification performed in the last column is described and used in section 3. 


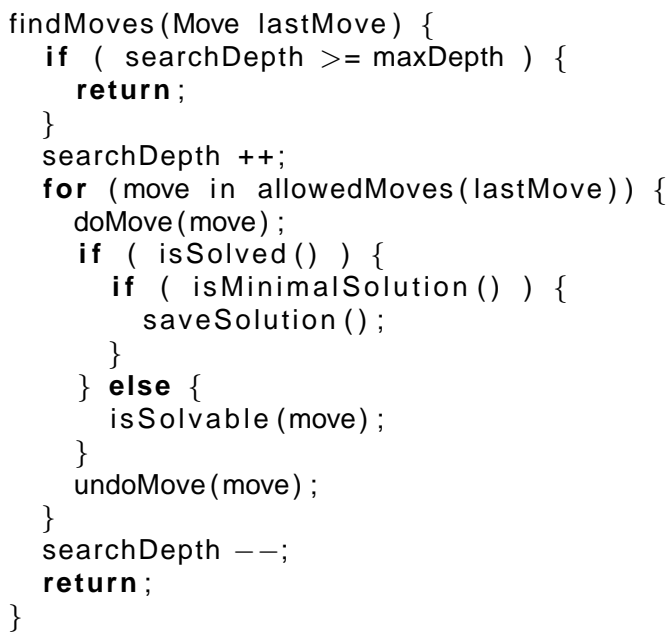

FIGURE 1: Algorithm for Shuffle Puzzle

\begin{tabular}{|c|c|c|}
\hline Label & Description & Classification \\
\hline NoStaticFunc & Static functions removed & Design \\
\hline NoCopies & Recursion does no operate on copies any more & Design \\
\hline Redesign & Complete redesign of solution & Design \\
\hline DataType & Data type in breadth-fi rst search changed & Design \\
\hline Copies & Recursion operates on copies & Design \\
\hline Visibility & Visibility of variables changed & Design \\
\hline NoStaticVars & Search does not operate on static variables any more & Design \\
\hline WrongFormat & Output has wrong format & Specifi cation \\
\hline WrongSig & Signature of interface changed & Specifi cation \\
\hline Runtime & Search was too slow & Specifi cation \\
\hline TimeConstraints & Search fi nishes after 15 seconds and returns false & Specifi cation \\
\hline WrongSolution & Solution was wrong for solved puzzles as input & Specifi cation \\
\hline Thrashing & Solution contained up-down/left-right move & Specifi cation \\
\hline NotMinimal & Provided solution was not minimal & Specifi cation \\
\hline WrongActualDepth & actual search depth not tracked $(5,17)$ & Algorithm \\
\hline WrongBacktrack & actual search depth decreased two times (17) & Algorithm \\
\hline Nolnit & Empty fi eld of shuffe puzzle not searched initially & Algorithm \\
\hline NoDeepCopy & No deep copy of fi rst puzzle & Algorithm \\
\hline WrongSolveCond & Wrong algorithm for isSolved (8) & Algorithm \\
\hline NoDeepComp & References compared instead of deep comparison (6) & Algorithm \\
\hline WrongDistance & Distance from solution wrong & Algorithm \\
\hline TooDeep & Search was too deep & Expression \\
\hline WrongBaseCond & Recursion does not terminate correctly (2) & Expression \\
\hline Wronglnit & Wrong initial value for actual search depth & Expression \\
\hline WrongMatrixIndex & Shuffle puzzle index calculation wrong & Expression \\
\hline Wronglndex & Index calculation for empty piece wrong & Expression \\
\hline WrongHistSize & Allocated space for data too small & Expression \\
\hline WrongMoveDir & Directions up-down/left-right mixed up (6) & Expression \\
\hline WrongSolLength & Solution has to be smaller than maximum search depth (9) & Expression \\
\hline ExpThrashing & Wrong condition for recognition of thrashing (6) & Expression \\
\hline WrongOutOfBoundsCond & Wrong identifi cation of matrix bounds (6) & Expression \\
\hline
\end{tabular}

\subsection{Polynomial}

The task of the problem Polynomial was to find the roots of an arbitrary polynomial of third degree. The participants had to implement the method getZeroPositions of the class Polynomial. The task description provided a hint for a possible algorithmic solution. The participants were pointed to the extreme values of the derivate of the polynomial in question and an estimation of a range where all roots of the polynomial had to lie. The given range should be divided into intervals with bounds given by the extreme values and the bounds of the range. The hint was to search for possible roots within these intervals with some fast search algorithm, for example the bisection method. Other search algorithms could be used as well. The problem of the Polynomial task was to identify 
the special cases that could occur. For example, not every polynomial of third degree has three roots, or, an extreme value can be a root as well.

Figure 2 shows a possible algorithm. First, the extreme values have to be calculated (line 2).

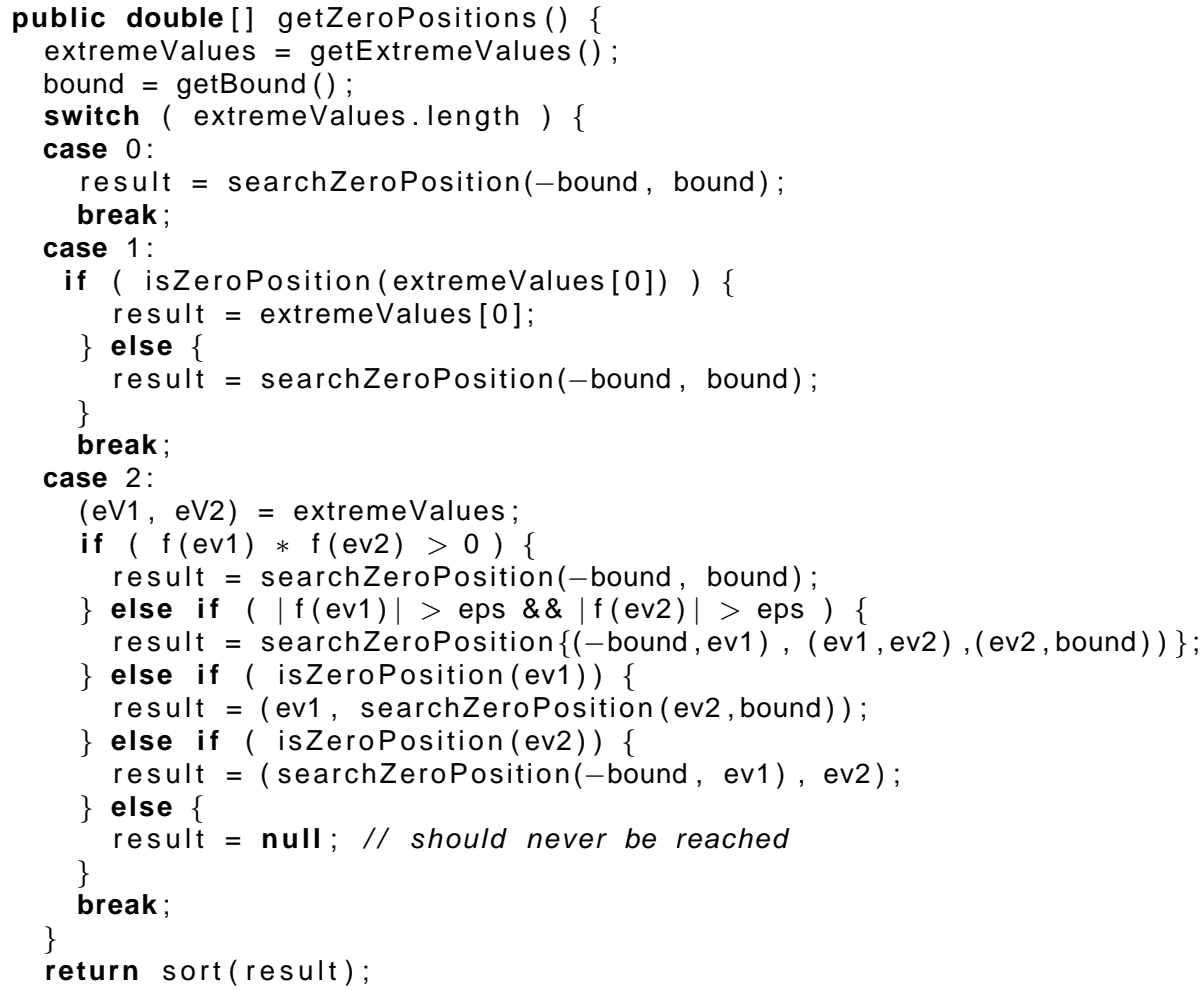

FIGURE 2: Algorithm for Polynomial task.

The quadratic formula was provided by the task description. Next, the search range has to be estimated. The Gershgorin Circle Theorem [12] was provided for this purpose. Afterwards, the implementation had to take into account the cases that the polynomials can have one (lines 5-7), two (lines 8-14), or three (lines 15-28) roots. The method isZeroPosition (lines 9, 21, 23) identifies whether a given value presents a root or not. The method searchZeroPosition (lines 6, 12, 18, $20,22,24)$ searches for a root for each provided interval. The search includes for example the selection of a proper start value which is essential for Newton's method. Finally, the roots should be sorted in ascending order (line 30). The combination of the special case of an extreme value which is also a root and the floating point arithmetic was very hard to solve.

Table 3 presents the classification of the defects found in programs of the Polynomial task. And again, the numbers in braces point a location in the algorithm and the classification in the last column is described in the next section.

\section{ANALYSIS OF DEFECTS}

We start the analysis of defects with an analysis of the number of defects caused by the pairs and the solo programmers. Figure 3 shows the number of defects caused by an experimental unit for each task. The defects of the Shuffle Puzzle programs are shown on the left hand side while the defects of the Polynomial programs are shown on the right hand side. The plot on the left hand side shows the advantage of the programmer pairs while the right hand side does not. Literature suggests [13] that programmer pairs make fewer mistakes than solo programmers. We investigated this hypothesis with the one-sided Wilcoxon test on our data set. The hypothesis holds for the Shuffle Puzzle task ( $p=0.015)$ but not for the Polynomial task $(p=0.123)$. But, why is there a difference for the Shuffle Puzzle task and not for the Polynomial task, as well? 
TABLE 3: Classification of defects found for the Polynomial task.

\begin{tabular}{|c|c|c|}
\hline Label & Description & Classification \\
\hline Maintenance & Maintenance & Design \\
\hline DZP.ResultWrongSize & Result vector has wrong size & Specifi cation \\
\hline EPSILON.SignatureChanged & constant $\epsilon$ changed to static & Specifi cation \\
\hline Specifi cation.WPA & Polynomials of wrong degree accepted & Specifi cation \\
\hline Specifi cation.NS & Roots not sorted (30) & Specifi cation \\
\hline DZP.Missed & Double root not removed from solution & Algorithm \\
\hline IB.Forgotten & Missing analysis of interval bounds & Algorithm \\
\hline IV.Missed & Analysis of one interval missed & Algorithm \\
\hline MF.ENS & Extreme values of derivation not sorted (2) & Algorithm \\
\hline Newton.WSV & Start value of newton conceptually wrong $(6,12,18,20,22,24)$ & Algorithm \\
\hline BOUNDS.Wcalc & Bounds wrong calculated (3) & Algorithm \\
\hline SPECIALCASE.OZP & Sole root not found (8-14) & Algorithm \\
\hline IF.UsageWrong & Interface not used correctly & Algorithm \\
\hline ALG.WrongAccuracy & Algorithm does not calculate root with proper accuracy & Algorithm \\
\hline DZP.WrongComparison & Wrong comparison for removal of double root & Expression \\
\hline ZP.Wrongldent & Identifi cation of root wrong $(9,21,24)$ & Expression \\
\hline ZP.WrongldentIntervall & Identifi cation if root exists in interval wrong & Expression \\
\hline MF.DiscWrongCondition & Condition for discriminant wrong (2) & Expression \\
\hline MF.DiscWrongRange & Range of discriminant wrong (2) & Expression \\
\hline MF.WF & Operand of derivation wrong implemented (2) & Expression \\
\hline MF.WrongDerivation & Derivation in quadratic formula wrong (2) & Expression \\
\hline EPSILON.Changed & $\epsilon$ changed from $10^{-4}$ to $10^{-9}$ & Expression \\
\hline EPSILON.RCM & $f(x)==0$ instead of $|f(x)|<\epsilon$ & Expression \\
\hline Newton.WSVC & Start value of newton wrong $(6,12,18,20,22,24)$ & Expression \\
\hline Newton.ExitConditionWrong & Exit condition for newton wrong & Expression \\
\hline HS.ExitConditionWrong & Exit condition for bisection method wrong & Expression \\
\hline BOUNDS.WrongSorted & Sorting condition for intervall bounds wrong & Expression \\
\hline TYPO & Typo & Expression \\
\hline PD.WrongDegree & Division of polynomial with polynomial of degree zero & Expression \\
\hline
\end{tabular}
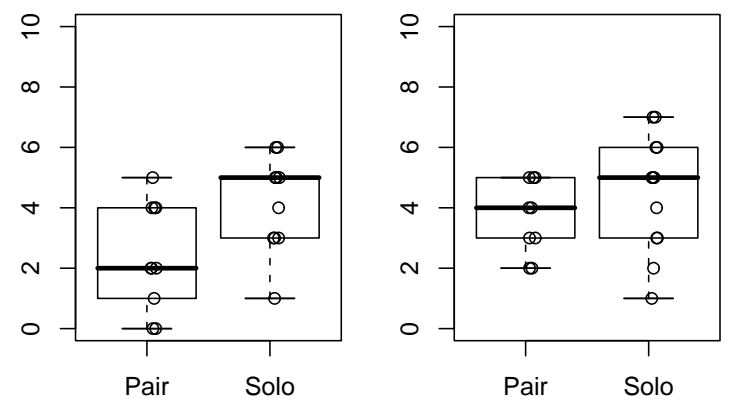

FIGURE 3: Comparison of number of defects for Shuffle Puzzle (left) and Polynomial (right).

To answer the question above, we classified each defect into one of the following categories.

Specification These defects violate some requirements stated in the specification. For example, a correct solution to the Polynomial tasks should report the roots in ascending order. If a program did not comply with this constrain, it was counted as having a specification defect.

Expression Defects categorised as expression defects are fixed by the correction of a single expression or condition.

Algorithm Algorithmic defects are removed by changing more than a single expression.

The last category are Design flaws. As opposed to the previous categories, design flaws are not defects, they are refactorings made during the acceptance-test phase to enhance program readability. We added this kind of program changes because we assumed that programmer pairs would not restructure their programs as often during the acceptance-test phase as the solo developers. The classification is presented in the last columns of Tables 2 and 3. 
Motivated by other studies [13], we assume that for each of the above categories programmer pairs cause fewer defects than solo programmers, and, that the advantage of the programmer pairs is evenly distributed on the above categories. Figure 4 shows for the Shuffle Puzzle and the Polynomial task and for each category the number of the defects. The plot of the left hand
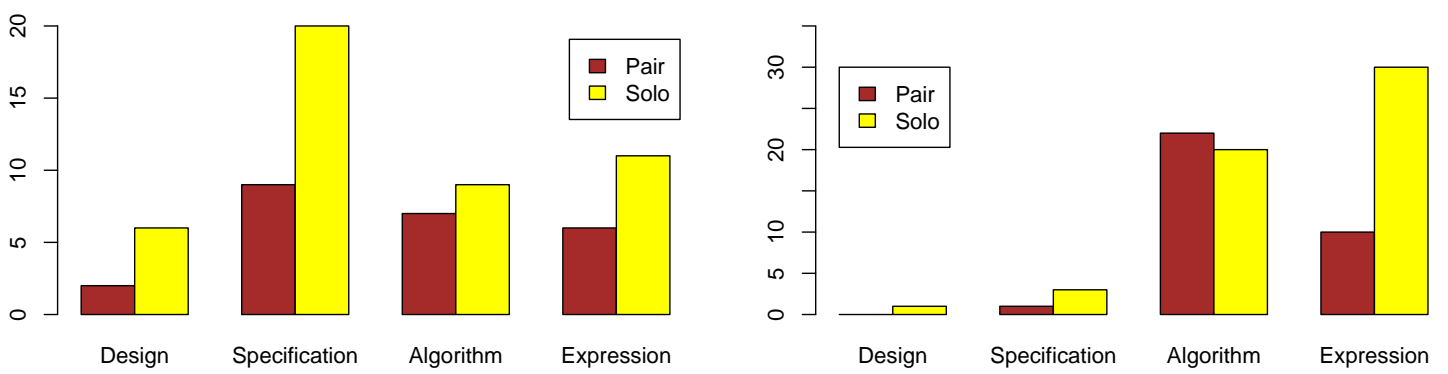

FIGURE 4: Classification of defects for Shuffle Puzzle (left) and Polynomial (right).

side of Figure 4 seems to support our expectation for the Shuffle Puzzle task. The bar of the solo programmers (the right bar in the figure) is larger for Design, Specification, Algorithm, and Expression which means that the solo programmers made more mistakes in all categories. However, the difference is smallest for the Algorithm defects. The difference is not that obvious for the Polynomial task, see the right plot of Figure 4. Here, the pairs outperform the solo programmers only for the Expression defects. Another observation concerns the relation of Algorithm to Expression defects. For Shuffle Puzzle, the pairs made only one Algorithm defect more than Expression defects. However for Polynomial, the number of Algorithm defects made by the pairs are more than twice as large as the Expression defects.

Table 4 shows the p-values of the one-sided Wilcoxon test which was performed on each of the above defect classes. Differences on a five percent level are highlighted with bold values. Defects

TABLE 4: Wilcoxon p-values of defect classification.

\begin{tabular}{l|cccc} 
Task & Design & Specification & Algorithm & Expression \\
\hline Shuffle Puzzle & 0.18 & $\mathbf{0 . 0 2}$ & 0.50 & 0.15 \\
Polynomial & 0.22 & 0.36 & 0.94 & $\mathbf{0 . 0 1}$
\end{tabular}

which can be fixed by correcting only a single expression seem to be reduced by pair programming regardless of the type of task. For Shuffle Puzzle, the pairs seem to make less restructurings than the solo developers, and the solutions of the pairs adhere more to the specification than the programs of the solo developers do. This last two findings do not hold for the Polynomial task.

The reason why programmer pairs made fewer mistakes in the Shuffle Puzzle task as compared to the Polynomial task could be explained by the reduced number of design flaws and specification violations. But the defect classification raises another question. Why is the relation of the pairs' Algorithm defects to Expression defects larger for Polynomial than for Shuffle Puzzle? To answer this question we investigate the detailed defect distribution of the pairs and solo programmers. Figures 5 and 6 show the detailed distribution for the Shuffle Puzzle and Polynomial task, respectively. Tables 5 and 6 in the appendix provide the complete data set.

The bar plot in Figure 5 reveals that there are 12 mistakes in the Shuffle Puzzle implementations that were made by more than one pair or one solo programmer. From these 12 defects, NoStaticVars belongs to Design. The 5 defects starting with WrongFormat up to NotMinimal belong to Specification. Three defects belong to Algorithm (WrongActualDepth, WrongSolveCond, WrongDistance). The remaining 3 defects WrongBaseCond, WrongMatrixIndex, and WrongMoveDir are Expression defects. All other defects might be seen as noise. It seems as if the complexity of the Shuffle Puzzle was the compliance to the specification. 


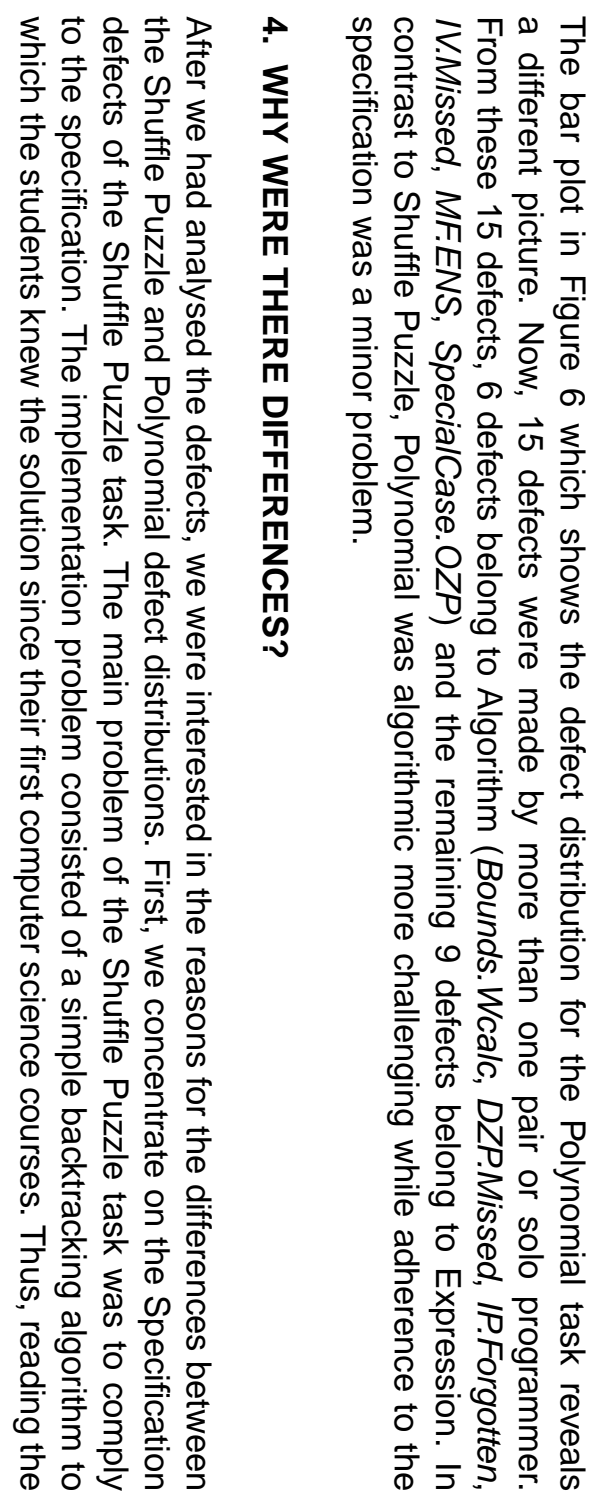
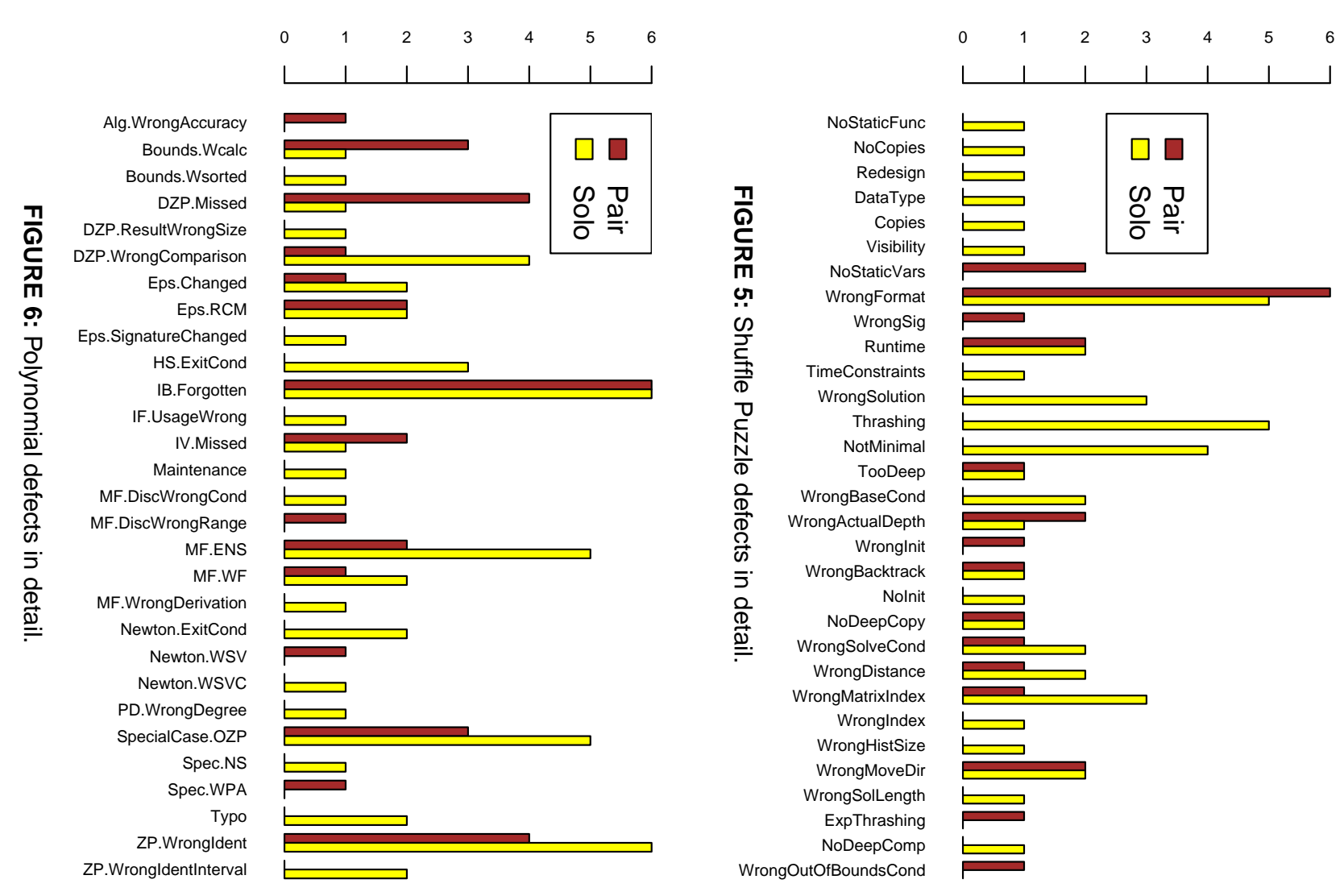
task description could have lead to premature conclusions what the task is about. The reason why pairs made fewer Specification defects could be their larger collective memory [1, p. 332] which reduces the chance that the pair misses a requirement stated in the specification as compared to a solo programmer. However, the larger collective memory did not prevent the pairs from violating the output format. Six (6) pairs violated the specification of the output format while only 5 solo developers did not comply to this requirement as well, see defect WrongFormat.

The next topic focuses on the more algorithmic defects in the Polynomial programs as compared to the Shuffle Puzzle programs. The Polynomial task was more complicated than the Shuffle Puzzle task. For example, the defect IB.Forgotten presented by Figure 6 was made by 6 independent solo developers and by 6 independent pairs. The fact that interval bounds have to be inspected in order to calculate the roots correctly is hard to identify. Literature reports on problems which are hard to solve regardless of the used methodology [4]. Therefore, it is reasonable that these problems are hard to solve for programmer pairs, as well.

The last topic deals with the observation that there are defects apart from the above discussed Specification defects which were introduced by fewer pairs than solo programmers. This difference can be partially explained with eureka problems from the social psychology. For an eureka problem, an individual who solves it will ordinarily feel quite certain that he is right [10, pp. 19]. A typical eureka problem is for example the following one:

On one side of a river are three wives and three husbands. All of the men but none of the woman can row. Get them all across the river by means of a boat carrying only three at one time. No man will allow his wife to be in the presence of another man unless he is also there [10, p. 19].

The correctness of the proper solution to this wives and husbands problem is readily apparent or easily demonstrated once that solution has been obtained. This characteristic of eureka problems leads to the observation that groups find the right answer unless one of its individuals possesses the ability to solve it. Thus, if the probability that one individual from a group of individuals fails to solve the problems is $Q$, the probability that a group made up of two individuals fails to solve the problem as well reduces to $Q^{2}$. This effect can be seen at its best for MF.ENS defect. MF.ENS states that the participants missed to sort the extreme values obtained by the quadratic formula. If you are pointed to the solution, that sorted values are necessary for a correct calculation of the interval bounds, you will be convinced directly. Thus, MFENS is an eureka problem. From the 12 solo programmers 11 had the chance to make this mistake because one solo programmer used a different algorithm. Now, 5 out of 11 developers made this error, see Figure 6 , leading to a theoretical failure probability for the pairs of $(5 / 11)^{2}=0.21$ which is comparable to the actual failure probability of $2 / 9=0.22$. Similar values can be calculated for another eureka problem, for example WrongMatrixIndex in Shuffle Puzzle with $(3 / 11)^{2}=0.07$ and $1 / 10=0.1$.

The discussion of the effects of eureka problems is interesting as it points to problem domains where the usage of programmer pairs instead of solo programmers might lead to a reduced risk of failure. For example, if a technological problem has to be solved and the solution can easily identified as the right one, the usage of a programmer pair does not necessarily mean nearly doubled personnel cost but rather a reduced probability of failure.

However, there still exist some Algorithm and Expression defects where the number of pairs who inserted this defect exceeds the number of solo developers, e.g. Bounds.Wcalc, DZP.Missed, IV.Missed, and WrongActualDepth. We do not know so far whether these failures are only due to chance or whether they expose another characteristic of the pair programming situation.

\section{THREATS TO VALIDITY}

Defect identification was done by humans. The defects of both problems were identified by one person. The identified defects together with their defect description were then reviewed by a second person. Inconsistencies or conflicts where resolved in a follow up discussion. Classification was straight forward and followed the rules presented in 3 . 
Participants where students and the pairs were ad-hoc pairs. Thus, the presented figures might be different if professionals and harmonising pairs had been used instead.

\section{CONCLUSIONS}

This paper presented the comparison of defects caused by pair and solo programmers. We assumed that programmer pairs make fewer mistakes than solo programmers regardless of the programming task and the defect type. However, our data set suggests that our assumption does not hold in general:

- Programmer pairs made as many algorithmic mistakes as solo programmers.

- The programs of the pairs had fewer faulty expression defects.

- For eureka problems, the number of pairs failing to solve the problem correctly could be approximated by the number of solo programmers who failed to solve the problem as well.

The first and the second result confirm the observation made by other studies [3] where pair programming was seen most productive for simple problems in simple code. The third result is interesting in terms of risk reduction. For challenging problems where a solution can easily be identified as the right one the usage of a programmer pair instead of a single developer might be a better choice.

Further studies should compare the character and difficulty of the programming assignments of previous pair programming studies. This investigation might clarify the still divergent picture on the productivity of pair programming as compared to solo programming.

\section{ACKNOWLEDGEMENTS}

The author would like to thank Rafael Sánchez-Moreno for inspecting the Polynomial programs, Andreas Höfer for the helpful discussions and his review of the Shuffle Puzzle programs, and Guido Malpohl for proof reading a previous version of this article.

\section{REFERENCES}

[1] D. Forsyth. Group Dynamics. Thomson Wadsworth, 4th edition, 2006.

[2] S. Heiberg, U. Puus, P. Salumaa, and A. Seeba. Pair-programming effect on developers productivity. In XP 2003, number 2675 in LNCS, pages 215-224. Springer, 2003.

[3] H. Hulkko and P. Abrahamsson. A multiple case study on the impact of pair programming on product quality. In International Conference on Software Engineering, pages 495-503, St. Louis, Missouri, USA, May 2005.

[4] C. Knight and N. Leveson. An experimental evaluation of the assumption of independence in multi-version programming. IEEE Transactions on Software Engineering, 12(1):96-109, 1986.

[5] M. Müller. Are reviews an alternative to pair Programming? Journal on Empirical Software Engineering, 9(4):335-351, December 2004.

[6] M. Müller, J. Link, R. Sand, and G. Malpohl. Extreme programming in curriculum: Experiences from academia and industry. In Conference on Extreme Programming and Agile Processes in Software Engineering (XP2004), pages 294-302, Garmisch-Partenkirchen, Germany, June 2004.

[7] M. Müller. Two controlled experiments concerning the comparison of pair programming to peer review. Journal of Systems and Software, 78:166-179, 2005.

[8] J. Nawrocki and A. Wojciechowski. Experimental evaluation of pair programming. In European Software Control and Metrics (Escom), London, UK, 2001.

[9] J. Nosek. The case for collaborative programming. Communications of the ACM, 41(3):105108, March 1998.

[10] I. Steiner. Group Process and Productivity. Academic Press, 1972.

[11] J. Tomayko. A comparison of pair programming to inspections for software defect reduction. Computer Science Education, 12(3):213-222, 2002. 
[12] E. Weisstein. Gershgorin circle theorem. From MathWorld-A Wolfram Web Resource. http://mathworld.wolfram.com/GershgorinCircleTheorem.html.

[13] L. Williams, R. Kessler, W. Cunningham, and R. Jeffries. Strengthening the case for pairprogramming. IEEE Software, pages 19-25, July/August 2000.

\section{A. ORIGINAL DATA SETS}

TABLE 5: Errors made by experiment unit for the Shuffle Puzzle task ( $P=$ pair, $S=$ =solo).

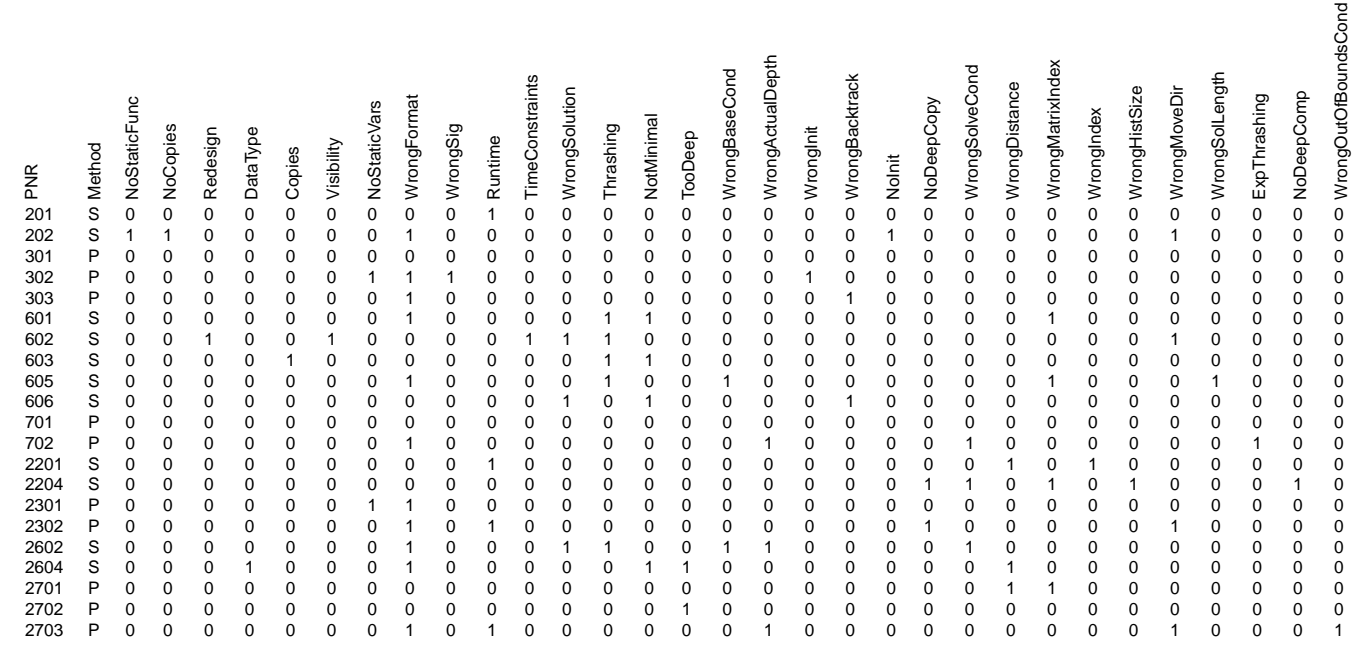

TABLE 6: Errors made by experiment unit for the Polynomial task ( $P=$ pair, $S=$ solo).

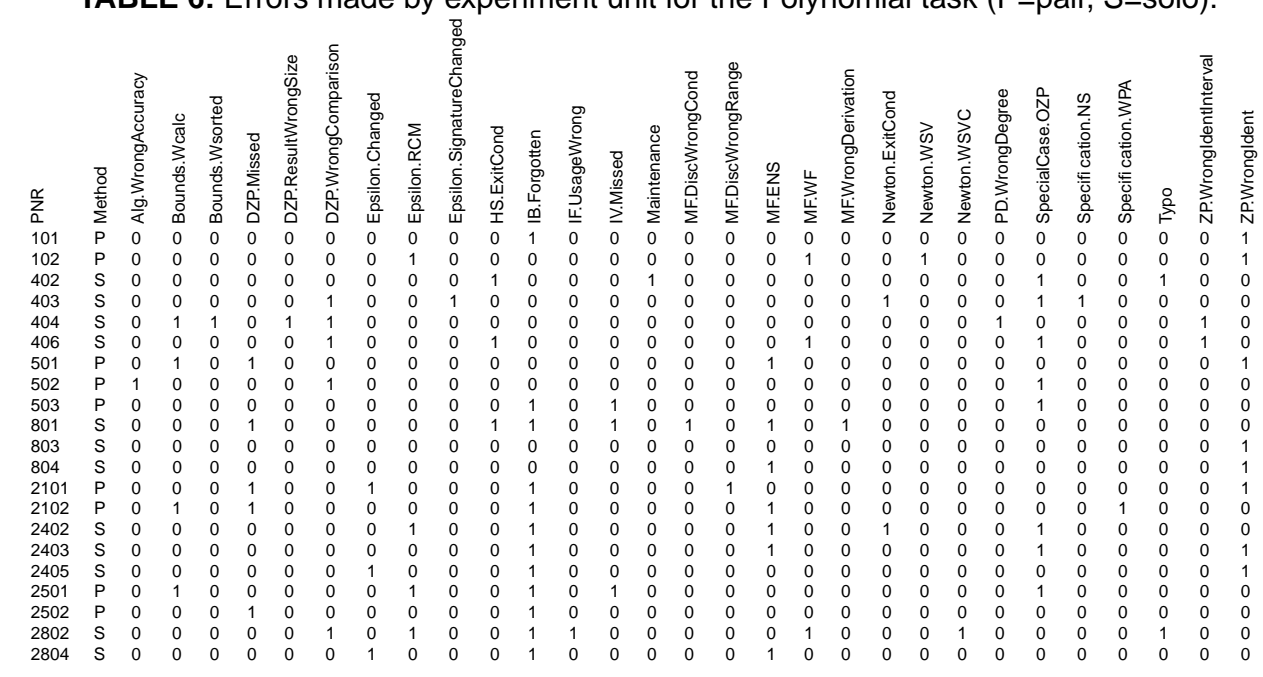

